\title{
Determinación de Periodos Fisiológicos en la Maduración y Calidad del Aceite de Piñón Blanco (Jatropha curcas L.)
}

\author{
Richer Garay ${ }^{(1)}$, Edison Hidalgo ${ }^{(1)}$, Jhonny A. Alegría ${ }^{(1)(2)}$ y Oscar. W. Mendieta ${ }^{(2)}$ \\ (1) Instituto Nacional de Innovación Agraria (INIA), Estación Experimental "El Porvenir", \\ Unidad Postcosecha y Agroindustria; Carretera Fernando Belaunde Terry Km.13.5, \\ distrito de Juan Guerra, San Martín - Perú. \\ (2) Universidad Nacional de San Martín, Facultad de Ingeniería Agroindustrial, Jr. Amorarca s/n, \\ Tarapoto, Perú. (e-mail: rgaray@inia.gob.pe; ehidalgo@inia.gob.pe; jhaas_17@hotmail.com; \\ oscarment@hotmail.com)
}

Recibido Oct. 20, 2011; Aceptado Dic. 06, 2011; Versión final recibida Feb. 07, 2012

\section{Resumen}

El objetivo de este trabajo fue determinar el momento óptimo de cosecha de los frutos y los parámetros de calidad del aceite de piñón blanco (Jatropha curcas L), adecuados para la producción de biodiesel. El trabajo fue desarrollado en la Parcela Experimental El Porvenir del Instituto Nacional de Innovación Agraria del Perú. Se evaluó las características físicas de los frutos y de las semillas y las características químicas del aceite. Los frutos de piñón utilizados fueron seleccionados al inicio del fructificación. El estado de maduración R2 tuvo una duración de $29 \pm 1.8$ horas, el estado R3 de $12 \pm 1.6$ horas, el estado R4 $27 \pm 1.7$ horas y finalmente R5 de $24 \pm 1.0$ horas. El análisis químico mostró que las semillas de piñón contienen en el estado fisiológico R4 un $51.5 \%$ de grasa, mientras que el índice de acidez más elevado se encontró en el R6 con 3.01 $\mathrm{mgKOH} / \mathrm{g}$ aceite. Los frutos de piñón se pueden cosechar en los estados fisiológicos R3, R4 y R5 por contar con elevado contenido de aceite y bajo índice de acidez.

Palabras clave: piñón, Jatropha curcas L, estado fisiológico, tiempo de cosecha, biodiesel

\section{Determination of Physiological Periods in the Maturation and Quality Oil of Physic nut (Jatropha curcas L.)}

\begin{abstract}
The objective of this work was to determine the optimum time for fruit harvest and the quality parameters of physic nuts (Jatropha curcas $L$ ) suitable for the production of biodiesel. This work was developed in the experimental station El Porvenir of Instituto Nacional de Innovación Agraria in Perú. The physical characteristics of the fruits and seeds and the chemical characteristics of the oil were evaluated. The fruits used were selected at the beginning of fruiting. The R2 maturation status lasted $29 \pm 1.8$ hours, the state R3 $12 \pm 1.6$ hours, the state R4 $27 \pm 1.7$ hours and finally R5 $24 \pm 1.0$ hours. Chemical analysis showed that the seeds of physic nuts contain $51.5 \%$ fat at the physiological state R4 while the highest acidity was found in the R6 with $1.3 \mathrm{mg} \mathrm{KOH} / \mathrm{g}$ oil. The fruit of physic nuts can be harvested during the physiological states R3, R4 and R5 to have high oil content and low acidity.
\end{abstract}

Keywords: physic nut, Jatropha curcas L, physiological status, harvest time, biodiesel 


\section{INTRODUCCIÓN}

El piñón blanco (Jatropha curcas L.), es considerado como una alternativa potencial para la producción de biodiesel en diversas partes del mundo, pero poco se sabe sobre su sistema de producción, productividad, costos de producción, la incidencia de las plagas, la fisiología, la composición de las semillas y los procesos de extracción de aceite (Arruda et al., 2004).

La primera cosecha ocurre desde el primer año, tres meses después de la floración. Inicialmente pequeña, va aumentando luego de sucesivas cosechas hasta estabilizarse entre el quinto y el sexto año. Las frutas están listas para cosecharse 90 días después de la floración cuando los frutos alcanzan la coloración amarilla y las semillas han alcanzado su máximo desarrollo (Alfonso, 2008).

Los rangos de producción de semilla son de aproximadamente 0.4 toneladas por hectárea por año hasta por encima de 12 t/há. Los frutos de piñón son cápsulas drupáceas y ovoides, inicialmente verdes pero variando a café oscuro o negro con el tiempo. Las cápsulas de los frutos son de $2.5 \mathrm{a}$ 4.0 centímetros de largo por 2.0 centímetros de ancho, elipsoidales y lisas que cuando maduran van cambiando a amarillas. Al inicio son carnosas pero dehiscentes cuando son secas. El desarrollo del fruto necesita 90 días desde la floración hasta que madura la semilla (Mejía, 2006). En la Fig. 1 se muestran frutos de piñón en estado verde.

Uno de los mayores obstáculos para viabilizar el cultivo es la floración discontinua, con frutas en la misma inflorescencia y diferentes edades, lo que implica la no maduración homogéneas y las dificultades en la cosecha (Saturnino et al., 2005).

La variabilidad del rendimiento de aceite de J. curcas es considerablemente alta, incluso para los frutos del mismo color, que a pesar de la rápida acumulación de aceite en la maduración, la humedad es bastante alta, incluso para las muestras que se han secado en el árbol. La separación de la pulpa del fruto y el secado de la semilla debe hacerse rápidamente para evitar el aumento de la acidez del aceite. La variación del contenido de aceite de las muestras de semillas de los frutos de color verde amarillo, amarillo oscuro a marrón era el mismo, lo que indica una gran variabilidad, del 20.0 al $43.0 \%$ en base seca (Frauches et al., 2011).

En relación a la acidez, se trata de un análisis que no diferencian entre ácidos orgánicos y ácidos grasos libres, provenientes de la hidrólisis de los triglicéridos. La acidez cuantificada para las muestras de color verde es elevada probablemente debido a la presencia de ácidos orgánicos o sustancias titulables con $\mathrm{NaOH}$, la cual se va reduciendo a medida que el fruto madura. En las muestras de fruto marrón seco, la acidez del aceite se ve probablemente a la presencia de ácidos grasos libres, debido a la alta humedad. La acidez de 1.2 a $6.0 \mathrm{mg} \mathrm{KOH} / \mathrm{g}$, significaría de 0.6 a 3.0\% de ácidos grasos libres (calculada como ácido oleico). La acidez se debe a la hidrólisis de los triglicéridos por lipasas, en virtud a los procesos de germinación, en condiciones de alta humedad de la semilla (Frauches et al., 2011).

La maduración del fruto de J. curcas ocurre durante un período largo, lo que requiere una recolección semanal durante varias semanas o incluso varios meses al año. La maduración del fruto no es uniforme. Sólo los frutos maduros (de color amarillo, marrón y negro) pueden ser recolectados (Rijssenbeek, 2009).

La madurez se puede establecer a partir del momento en el que la fruta comienza a tornarse amarilla pasando en el proceso del color verde al amarillo-verdoso. Las pruebas realizadas en distintas partes del mundo establecen que los frutos color verde tienen semillas con embriones en proceso de formación, que no son adecuadas para propagación aunque es posible una mínima extracción de aceite (Alfonso y Reyes, 2010).

Los frutos producen tres almendras negras, cada una de aproximadamente 2.0 centímetros de largo y 1.0 centímetro de diámetro; en promedio, el peso de 1000 semillas es aproximadamente 500 g. Las semillas contienen entre $50-60 \%$ de aceite, 30-32\% de proteína y 60-66\% de lípidos. 
La cáscara representa el $43 \%$ de la semilla y el grano $57 \%$, del cual el $30 \%$ es grasa cruda. El aceite contiene $21 \%$ de ácidos grasos saturados y $79 \%$ de ácidos insaturados. La semilla contiene minerales como fósforo, calcio, sodio, potasio y magnesio, y además algunas sustancias químicas nocivas para el ser humano como los ésteres de forbol (Mejía, 2006). Las semillas de piñón se muestran en la Fig. 1.

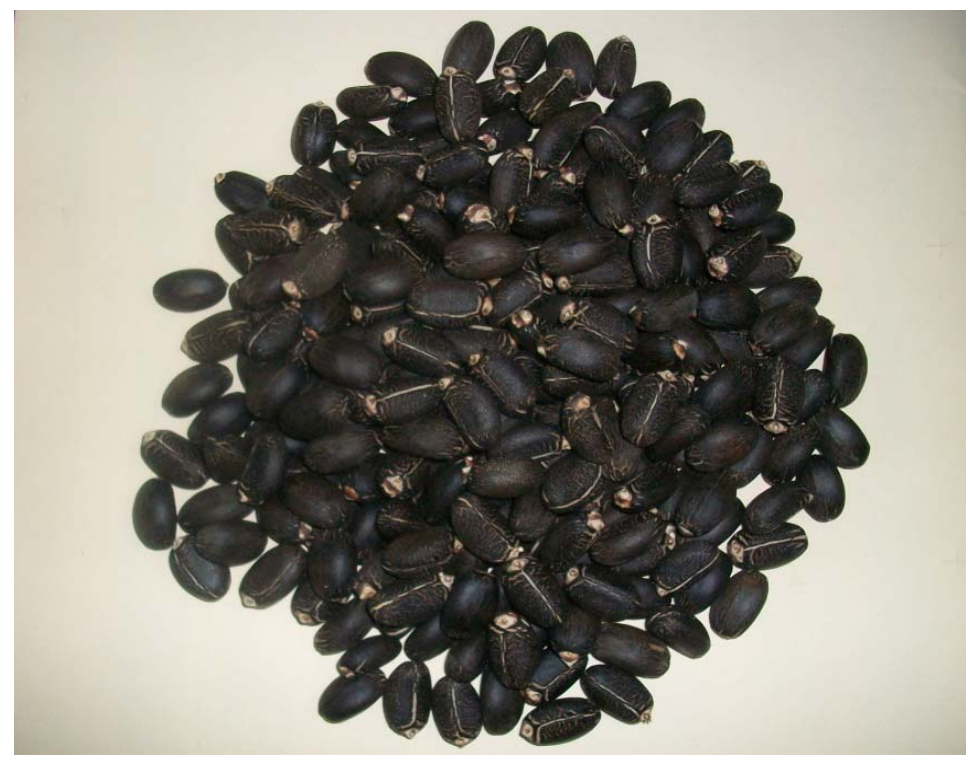

Fig. 1: Semillas de piñón

El objetivo del presente trabajo fue la determinación del momento óptimo de cosecha de los frutos y los parámetros de calidad del aceite de piñón blanco (Jatropha curcas L)

\section{MATERIALES Y MÉTODOS}

El estudio se realizo entre los meses de Octubre a Diciembre 2010 y Enero a Mayo 2011. La zona donde se realizo el estudio se encuentra ubicada en latitud sur de 06³6'15", longitud oeste $76^{\circ} 21^{\prime} 15^{\prime \prime}$ y altitud de 230 msnm. Los frutos evaluados fueron obtenidos de la parcela instalada en la Estación Experimental Agraria "El Porvenir" lote A1, perteneciente al genotipo Toctorillayco y de 04 años de edad. En la parcela la densidad de siembra fue de $2 \mathrm{~m}$. entre plantas y $3 \mathrm{~m}$. entre hileras. Los análisis fisicoquímicos se realizaron en el laboratorio de Postcosecha y Agroindustria, perteneciente al Instituto Nacional de Innovación Agraria (INIA), ubicada en el distrito de Juan Guerra, departamento de San Martín.

Los frutos de J. curcas utilizados en la presente investigación, fueron seleccionados al inicio del botón de fructificación. Se seleccionó al azar una muestra de 12 plantas, de una población inicial de 100 individuos, de las cuales se marcaron 5 frutos por planta, sumando en total 60 unidades experimentales inicialmente.

Las medidas de los diámetros y longitudes fueron tomadas con una regla milimetrada, para evitar el daño al fruto. Las evaluaciones se realizaron en intervalos de 2 y 3 días en los primeros 16 días, debido al rápido desarrollo de los frutos, y posteriormente cada 3 y 4 días ya que después de ese tiempo la tasa de crecimiento del fruto disminuye.

La definición de las escalas de evaluación por cada periodo de maduración se muestra en el Tabla 1. La escala presentada fue definida a partir de observaciones realizadas en las plantaciones del INIA y reportes tomados de la literatura (Alfonso y Reyes, 2010). 
Tabla 1. Escala de evaluación por cada periodo de maduración de J. curcas.

\begin{tabular}{|l|c|}
\hline \multicolumn{1}{|c|}{ Evento } & Escala \\
\hline Inicio de fructificación (2. 16 diámetro y 2.58 longitud) & $\mathrm{R}_{0}$ \\
\hline Frutos de color verde - semilla de color negra & $\mathrm{R}_{1}$ \\
\hline Frutos de color verde amarillo - pintón & $\mathrm{R}_{2}$ \\
\hline Frutos de color amarillo intenso & $\mathrm{R}_{3}$ \\
\hline Frutos de color amarillo - manchas marrones & $\mathrm{R}_{4}$ \\
\hline Frutos de color marrón - café & $\mathrm{R}_{5}$ \\
\hline Frutos de color gris & $\mathrm{R}_{6}$ \\
\hline
\end{tabular}

Elaboración propia.

El control del periodo de maduración se realizó 3 veces al día, a las 7:00 horas, 11:00 horas y 15:00 horas, observando el cambio de coloración del fruto.

Para la determinación de la calidad del aceite se recolectaron $15 \mathrm{~kg}$ frutos en cada estado de maduración de la misma parcela experimental, los cuales fueron sometidos a despulpado para retirar las semillas. Las semillas fueron secadas en estufa a $60^{\circ} \mathrm{C}$ por 8 horas, hasta alcanzar el $7 \%$ de humedad. Posteriormente se sometieron a prensado mecánico con una prensa de tornillo sin fin regulada a la velocidad de giro de 22 RPM (Prensa KEK - P0020), con previo funcionamiento trabajando en vacio por un tiempo de 15 minutos. El aceite bruto obtenido del proceso de extracción se filtró con papel filtro (WHATMAN N 40 de $125 \mathrm{~mm}$ de diámetro) y con ayuda de una bomba de vacío. El análisis de humedad de las semillas se realizó por el método de secado en estufa, a $105^{\circ} \mathrm{C}$ por 24 horas (AOAC, 1990); así mismo se determinó la grasa total por el método soxhlet (AOAC, 1990), utilizando como solvente éter de petróleo. Para la determinación del índice de acidez se utilizó el método de titulación con KOH a 0.1 N. (AOCS, 2005).

Se realizaron con la ayuda de un colorímetro digital (marca Minolta CR - 400), por parte de este instrumento se basa en los tres elementos primarios de los colores que son: color = a, luminosidad $=\mathrm{b}$ y saturación $=\mathrm{c}$. Con cuales se forma un sistema asignándole a cada elemento un valor numérico correspondiente $a L^{*} a^{*} b^{*}$ respectivamente, donde $L^{*}$ es la luminosidad y $a^{*}$ y $b^{*}$ son la saturación. El índice de color $\left(I C^{*}\right)$ fue calculado mediante la ecuación: $I C^{*}=a^{*}$. 1000/ L*. $b^{*}$ (Vignoni et al., 2006),

Se aplicó un diseño completamente al azar con 3 repeticiones, con un nivel de significancia de $\alpha=0.05$, realizándose el análisis de varianza respectivo, la estimación de la confiabilidad de los datos fue evaluada mediante el Coeficiente de Variación (CV). Así mismo se realizaron pruebas comparativas de medias de Tukey en función a los diámetros y longitud de las semillas en los días de evaluación y la calidad del aceite. Para esto se utilizó el paquete estadístico (SAS System for Windows V8).

Se tuvo como variables respuestas los parámetros de calidad como el contenido de aceite, acidez, humedad y coloración.

\section{RESULTADOS Y DISCUSIÓN}

El inicio de fructificación ocurre cuando el fruto está totalmente formado y comienza el desarrollo, las dimensiones iniciales indican $2.16 \pm 0.16 \mathrm{~mm}$ de diámetro y $2.58 \pm 0.24 \mathrm{~mm}$ de longitud. Al inicio, el crecimiento es rápido, duplicando su tamaño en dos días. Aproximadamente a los $36.8 \pm 0.77$ días de iniciado la fructificación el J. curcas alcanza el estado de maduración R2.

A partir de la determinación de color de los frutos de J. curcas como se muestra en la Fig. 2 y Fig. 3, se llegó a establecer que el tiempo que permanece el fruto en el estado de maduración R2 es de $28.96 \pm 1.77$ horas, R3 de $12.26 \pm 1.61$ horas, R4 de $27.14 \pm 1.65$ horas y R5 de $24 \pm 0.99$ horas. Por otro lado, el análisis de varianza indica que existe una diferencia altamente significativa entre los periodos medios de los estados de maduración fisiológica. La prueba de Tukey indicó que no 
existe diferencia significativa entre los periodos promedios de los estados de maduración R2 y R4, diferencia significativa entre los periodos promedios de los estados de maduración R4 y R5, y finalmente diferencia altamente significativa para las demás comparaciones, como puede verse en la Fig. 4.

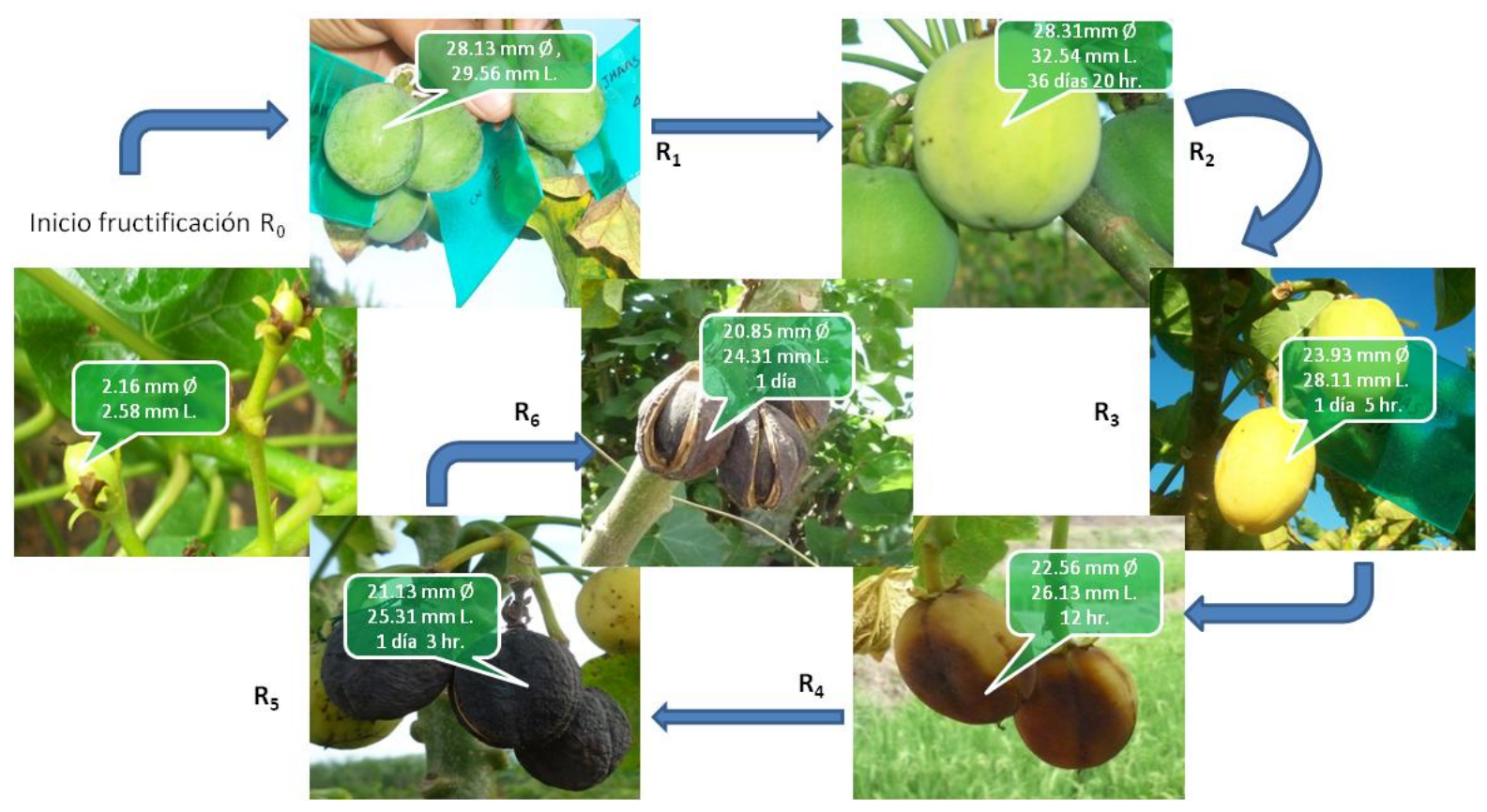

Fig. 2: Estados de maduración fisiológica de J. curcas en función al tiempo

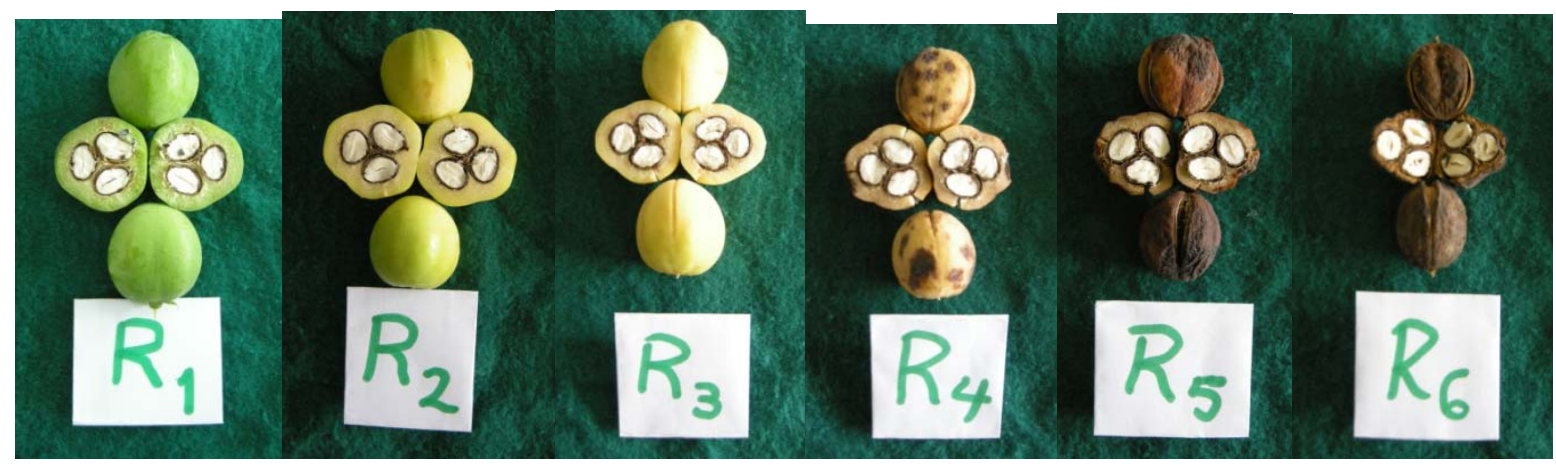

Fig.3: Corte de los frutos de J.curcas, en diferentes estados de maduración fisiológica

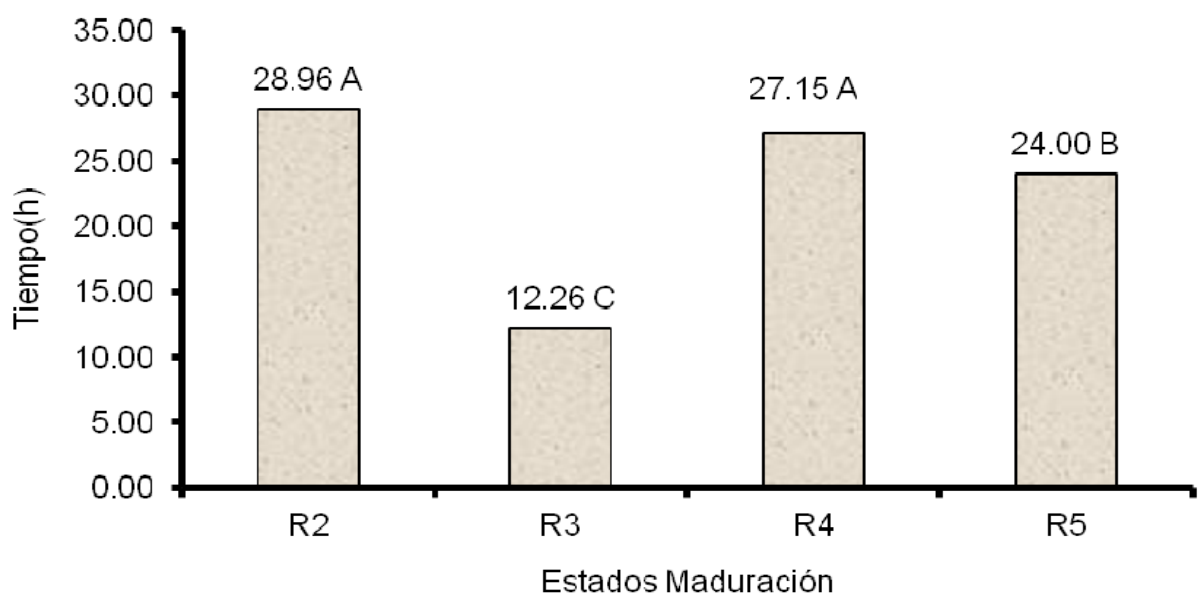

Fig. 4: Periodos de maduración fisiológica de J. curcas 
Las longitudes promedio para cada estado de maduración fisiológico fueron: R1 = 29.56 \pm 0.57 ; R2 $=32.54 \pm 0.49 ; \mathrm{R} 3=28.11 \pm 0.67 ; \mathrm{R} 4=26.13 \pm 0.66 ; \mathrm{R} 5=25.31 \pm 0.48 ; \mathrm{R} 6=24.31 \pm 0.58$, lãs cuales se presentan en La Fig. 5. El análisis de varianza indica que existe diferencia altamente significativa entre las medias de las longitudes de los estados de maduración fisiológica. El valor del coeficiente de variación fue de 3.03\%. La prueba de Tukey indicó que no existe diferencia significativa entre la longitud de un fruto en estado de maduración (R4 - R5), (R5 - R6), diferencia significativa entre (R1 -R3). Las demás comparaciones tuvieron diferencia altamente significativa.

Los diámetros promedio para cada estado de maduración fisiológica fueron: $R 1=28.23 \pm 0.25$; R2 $=28.31 \pm 0.96 ; \mathrm{R} 3=23.93 \pm 0.65 ; \mathrm{R} 4=22.56 \pm 0.96 ; \mathrm{R} 5=21.13 \pm 0.86 ; \mathrm{R} 6=20.85 \pm 0.59$, los cuales se muestran en la Fig. 5. El análisis de varianza indica que existe una diferencia altamente significativa entre las medias de los diámetros de los estados de maduración fisiológica. El coeficiente de variación presento un valor de $4.51 \%$. La prueba de Tukey indica que no existe diferencia significativa entre los diámetros de los estados de maduración fisiológica (R1 - R2), (R3 - R4), (R4 - R5) y (R5 - R6), las demás comparaciones tuvieron diferencia altamente significativa.

El desarrollo del fruto de J. curcas desde el inicio de fructificación hasta el estado de maduración fisiológica R6, se presenta en la Fig. 5. Este gráfico fue construido con la ayuda de los resultados anteriores. La curva muestra un comportamiento típico del desarrollo de todo fruto, inicia con una velocidad de crecimiento rápido, duplicando sus dimensiones en 2 días, para luego, pasado los 15 días un crecimiento lento y prolongado hasta los 36.82 días, momento en el cual comienza la etapa de pérdida de agua y el fruto comienza a perder también tamaño y forma.

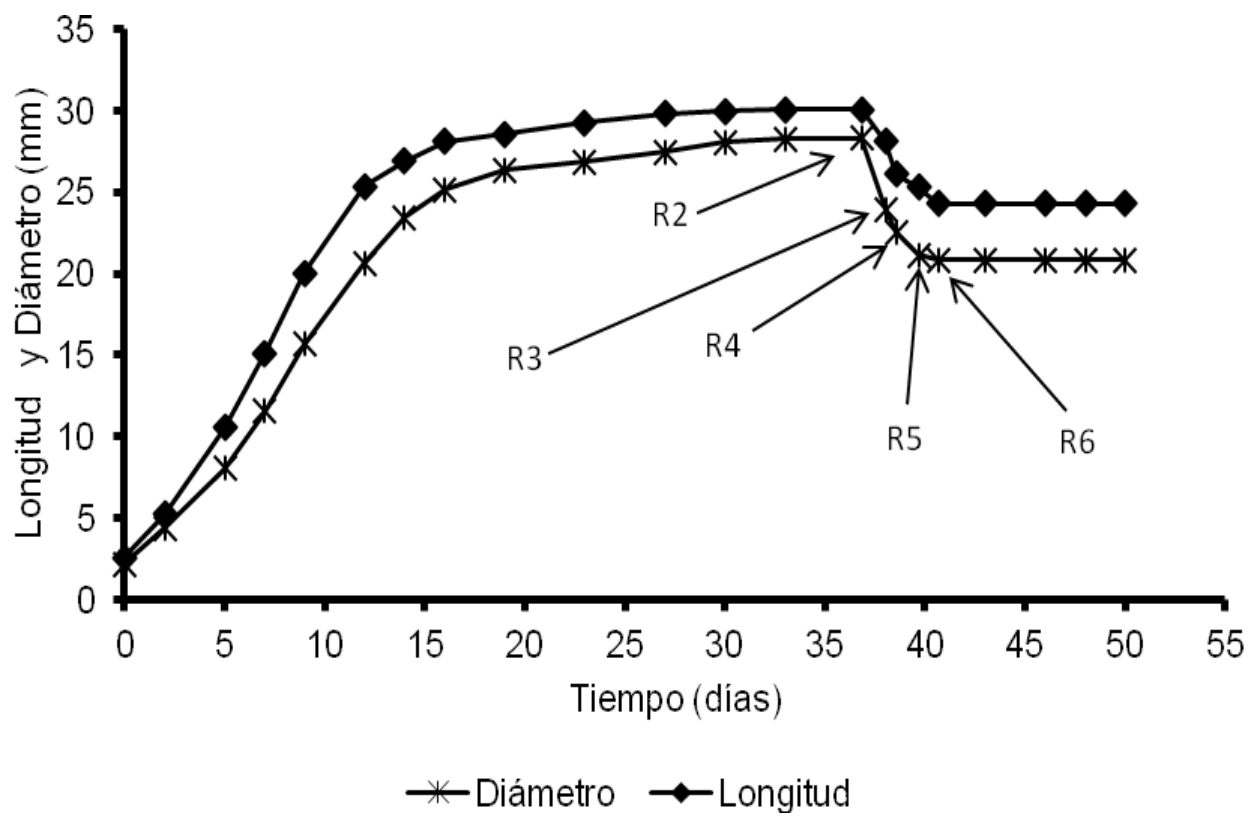

Fig. 5: Desarrollo del fruto de J. curcas

El estado de maduración R2 comienza a los 36.82 días de iniciado la fructificación y culmina a los 38.03 días, momento en el que comienza el estado R3 que permanece por solo unas horas culminando a los 38.54 días, el estado R4 perdura hasta el día 39.67, mientras que el estado R5 continua hasta el día 40.67, finalmente el estado R6 culmina cuanto el fruto se desprende, como se muestra en la Fig. 6. Según Mejía (2006) y Alfonso y Reyes (2010) el desarrollo del fruto necesita 90 días desde la floración hasta que madura la semilla, la semilla es cosechada cuando la cápsula está madura y cambia del verde a amarillo. Si tomamos este dato y considerando que la maduración a la que se refieren los autores mencionados es el estado R3, el periodo de floración de J. curcas dura 52 días. 


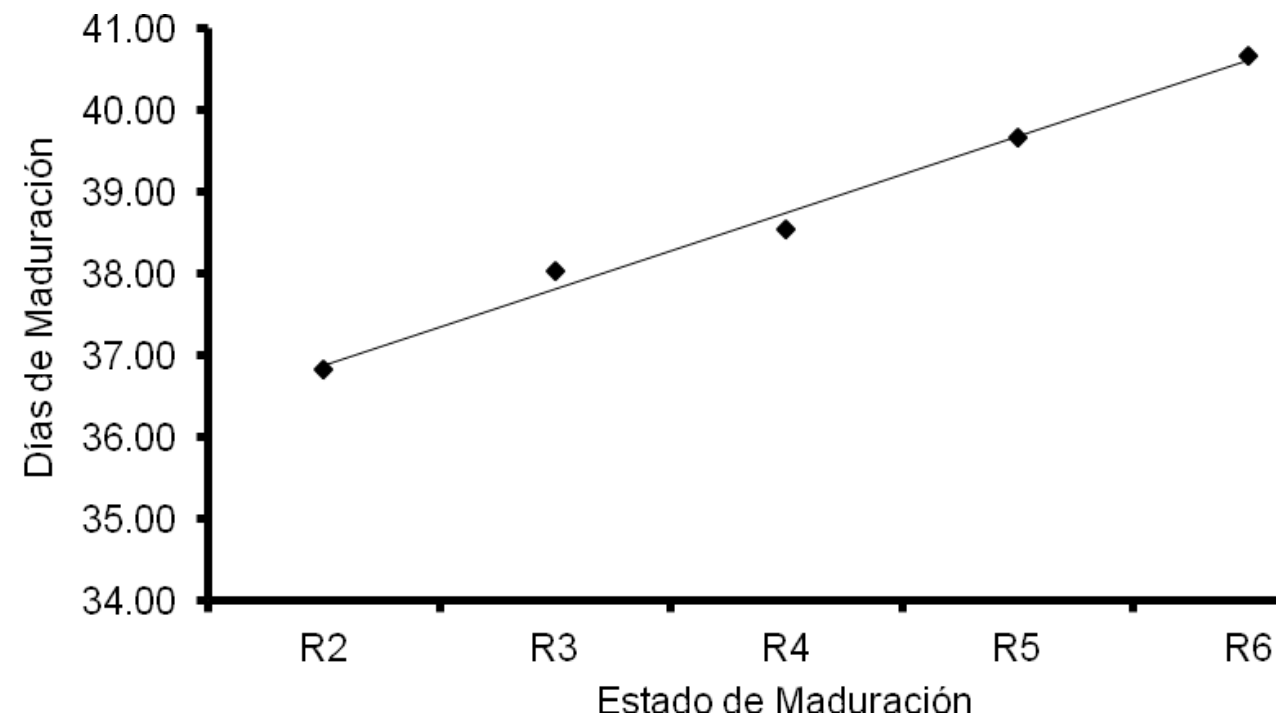

Fig. 6: Días de maduración del fruto de J. curcas después de iniciada la fructificación

Los contenidos de humedad en los diferentes estados fisiológicos se presentan en la Fig. 7. Puede notarse claramente la disminución en el contenido de humedad, desde el estado R1 hasta el estado R6, no habiendo diferencia significativa estadísticamente entre los estados R3 y R4 con contenidos de humedad de 44.62 y 44.23 , respectivamente como se muestra en la Tabla 2 . Los estados R3 y R4 presentaron diferencia estadísticamente significativa respecto a los demás estados fisiológicos (R1, R2, R5 y R6). Frauches et al. (2011), reportaron valores de humedad de $54 \%$ (verde oscuro), 38\% (amarillo oscuro) y 20\% (marrón), los cuales difieren de los resultados obtenidos en el presente trabajo, diferencia que puede atribuirse a los diferentes genotipos evaluados.

Tabla 2. Variación de los porcentajes de humedad $(\mathrm{PH})$, contenido aceite $(\mathrm{CA})$ e índice de acidez (IA) en diferentes estados fisiológicos de J. curcas.

\begin{tabular}{|c|c|c|c|}
\hline Estados & $\mathrm{PH}$ & $\mathrm{CA}$ & $\mathrm{IA}$ \\
\hline $\mathrm{R} 1$ & $51.07 \mathrm{a}$ & $48.28 \mathrm{c}$ & $1.11 \mathrm{~d}$ \\
\hline $\mathrm{R} 2$ & $49.94 \mathrm{~b}$ & $49.76 \mathrm{~b}$ & $1.28 \mathrm{~cd}$ \\
\hline $\mathrm{R} 3$ & $44.62 \mathrm{~b}$ & $51.19 \mathrm{a}$ & $1.44 \mathrm{cbd}$ \\
\hline $\mathrm{R} 4$ & $44.23 \mathrm{~b}$ & $51.53 \mathrm{a}$ & $1.52 \mathrm{cb}$ \\
\hline $\mathrm{R} 5$ & $38.49 \mathrm{c}$ & $51.27 \mathrm{a}$ & $1.72 \mathrm{~b}$ \\
\hline $\mathrm{R} 6$ & $9.98 \mathrm{~d}$ & $47.38 \mathrm{c}$ & $3.01 \mathrm{a}$ \\
\hline Media & 39.05 & 49.90 & 1.68 \\
\hline CV (\%) & 2.76 & 0.85 & 8.97 \\
\hline $\mathrm{R}^{2}$ & 99.6 & 95.79 & 96.51 \\
\hline
\end{tabular}

Medias seguidas de la misma letra en la columna no se diferencian por la prueba de Tukey al 5\% de probabilidad. 


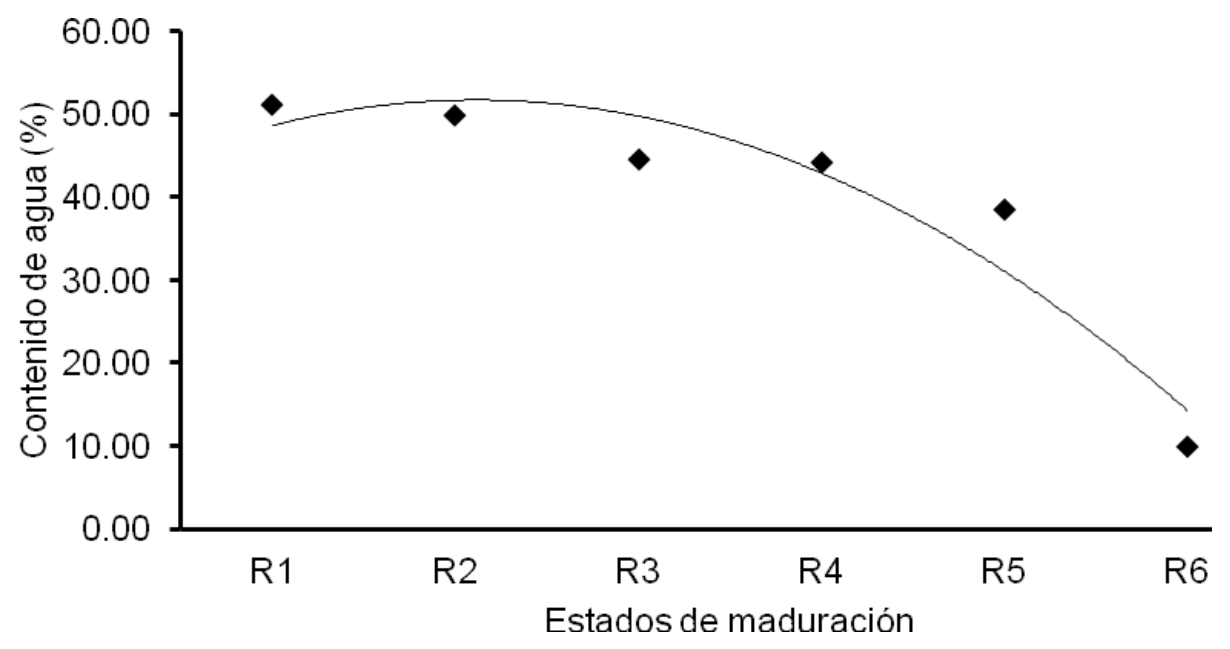

Fig. 7: Contenido de humedad en diferentes estados fisiológicos de J. curcas

Los resultados del contenido de aceite (\%), en los diferentes estados fisiológicos considerados, se presentan en la Fig. 8. Puede notarse que el mayor rendimiento se obtiene en el estado R4 (51.53\%), seguido por el estado R5 (51.27\%) y el estado R3 (51.19\%), no existiendo diferencia estadísticamente significativa entre sus valores promedio. Se presenta diferencia significativa respecto a los estados R1 y R2 y diferencia altamente significativa respecto del estado R6. Los resultados obtenidos permiten afirmar que si se requiere tener el mayor rendimiento de aceite, el estado R4 es el adecuado para la cosecha del fruto de piñón. Resultados reportados en la literatura (Frauches et al., 2011), presentaron una alta variabilidad en el contenido de aceite (base seca), para los diferentes estados de maduración; $26 \%$ (verde oscuro), 40\% (amarillo oscuro) y $43 \%$ (marrón), difiriendo con los resultados obtenidos en el presente trabajo lo cual se puede atribuir a los diferentes genotipos empleados.

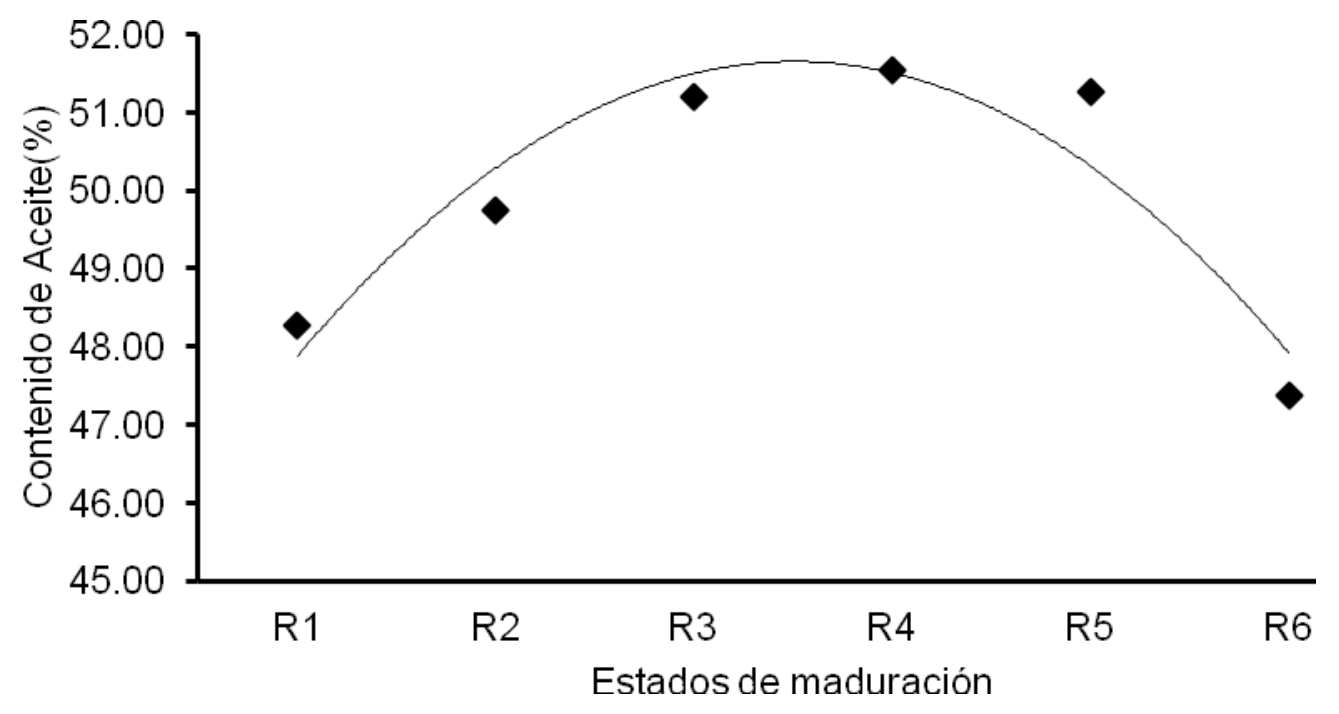

Fig. 8: Contenido de aceite de J. curcas en diferentes estados fisiológicos

Los resultados para el índice de acidez, que viene a ser un indicador de la calidad del aceite, se presentan en la Fig. 9. Puede verse que el mayor valor de acidez corresponde al estado R6, presentando diferencia significativa estadísticamente al determinado en los estados R4 y R5, y diferencia altamente significativa respecto a los estados R1, R2 y R3. Teniendo en cuenta que valores muy elevados de acidez en un aceite son un indicador de alto contenido de ácidos grasos libres y conllevan a tener baja calidad en los productos derivados de este aceite (biodiesel), se puede considerar al estado R5 como el adecuado para la cosecha del fruto, puesto que además es el estado en el cual se presenta el mayor rendimiento de aceite. Valores de índices de acidez 
obtenidos por Frauches et al. (2011), estuvieron comprendidos en el rango de 1.2 a $6.0 \mathrm{mgKOH} / \mathrm{g}$ de aceite para frutos de color marrón, equivalente al estado R5 cuyo índice de acidez fue de 1.52 $\mathrm{mgKOH} / \mathrm{g}$ aceite encontrarse en consecuencia en el rango reportado por los autores mencionados.

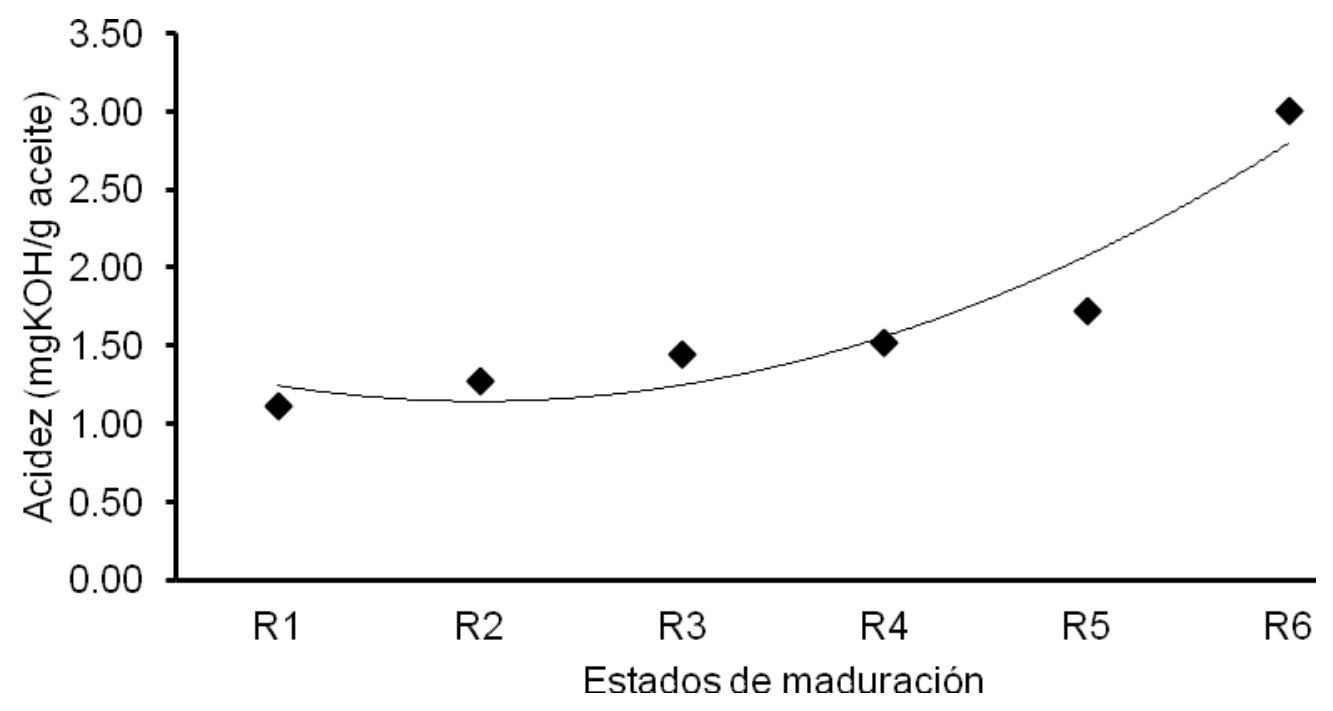

Fig. 9: Índice de acidez de J. curcas en diferentes estados fisiológicos

Adicionalmente, en la Fig. 10, se presenta la variación de parámetros de color $\left(L^{*}, a^{*}\right.$ y $\left.b^{\star}\right)$ de los frutos durante la maduración. Puede apreciarse que la luminosidad $\left(L^{*}\right)$ alcanza el valor más alto para el estado R3, y que esta característica se va incrementando desde R1 y R2 hasta R3 (aumento de la luminosidad relacionada con un color claro del fruto), para luego disminuir para R4, R5 y R6, estados en los cuales el color es más oscuro. En cuanto a los parámetros $a^{\star}$ y $b^{\star}$, sus valores van aumentando desde el estado R1 hasta el estado R3, disminuyendo luego hasta el estado R6; esta variación está relacionada con la pérdida del color verde del fruto, pasando a amarillo, amarillo café y negruzco en el estado R6, tal como se muestra en la Fig. 11, en la cual se presentan los índices de color para cada uno de los estados fisiológicos de piñón blanco.

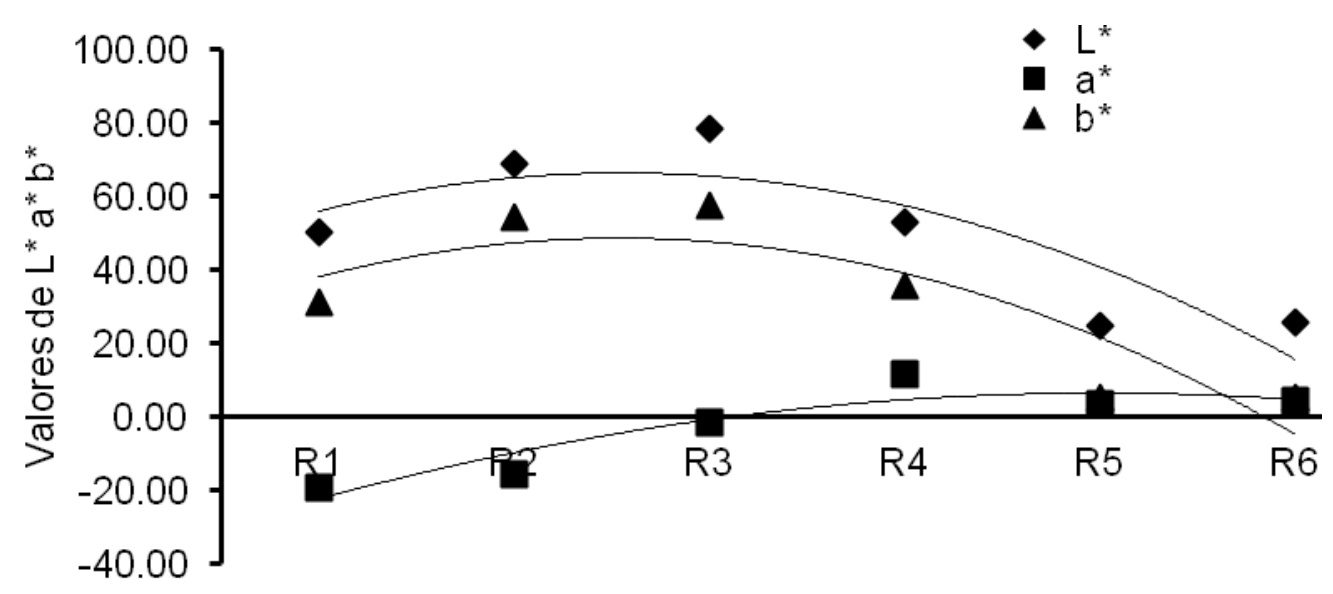

Estados fisiológicos

Fig. 10: Variación de parámetros de color durante la maduración de J. curcas en diferentes estados fisiológicos 


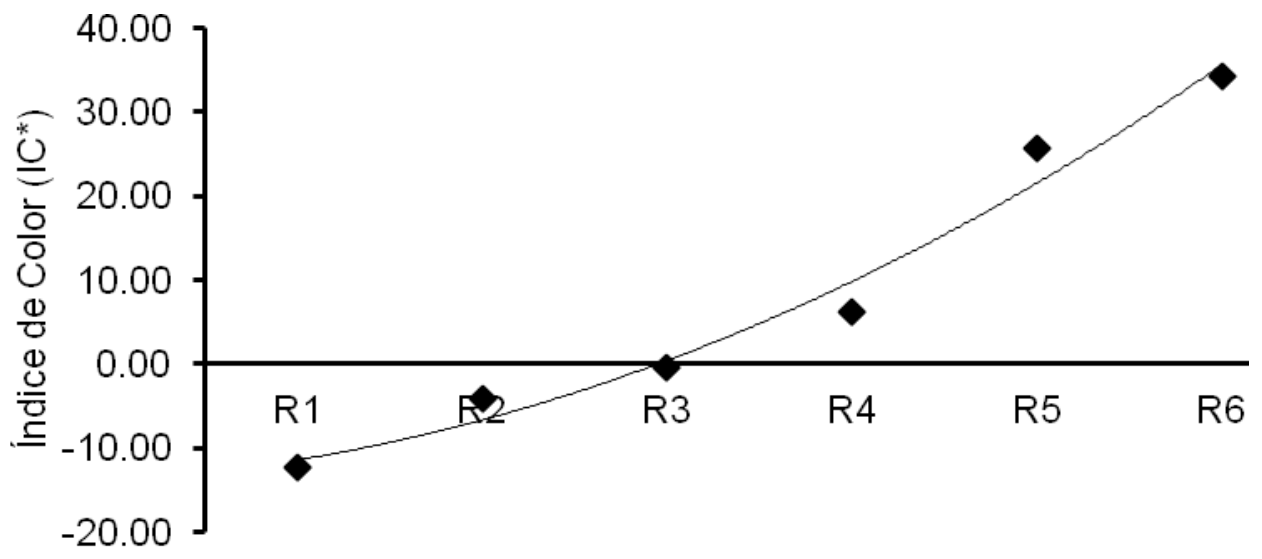

Estados fisiológicos

Fig. 11: Índice de color durante la maduración de J. curcas en diferentes estados fisiológicos

\section{CONCLUSIONES}

Se obtuvo diferencia altamente significativa entre los periodos medios de los estados de

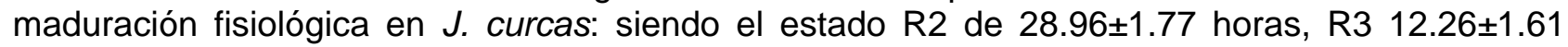
horas, R4 27.14 \pm 1.65 horas y R5 $24.00 \pm 0.99$ horas.

El estado de maduración R2 se origina a los 36.82 días de iniciada la fructificación y finaliza a los 38.03 días, luego inicia el R3 el cual permanece por solo unas horas, culminando a los 38.54 días; el estado R4 perdura hasta 39.68 días; el estado R5 continua hasta el día 40.67 y finalmente el estado R6 culmina cuanto el fruto cae.

El momento oportuno para realizar la cosecha de J. curcas es durante las etapas de maduración R3 al R5 ya que se obtienen el mayor contenido de aceite (51.53 a $51.19 \%)$, con bajo índice de acidez (< $1.52 \mathrm{mgKOH} / \mathrm{g}$ aceite).

A partir de las medidas de los parámetros de color $L^{*}, a^{*}$ y $b^{*}$ y del índice de color podría estimarse el estado fisiológico de los frutos, así como el contenido e índice de acidez del aceite de J. curcas.

\section{AGRADECIMIENTOS}

El presente trabajo se realizó con el financiamiento del Fondo de Innovación para la Ciencia y Tecnología - FINCyT contrato 030 Tarapoto, San Martín, República del Perú.

\section{REFERENCIAS}

Alfonso, J., Manual para el Cultivo de Piñon (Jatropha curcas) en Honduras. La Lima - Honduras. (2008).

Alfonso, J. y Reyes, P., Critérios para una Cosecha Eficiente y Selección de Semillas de Piñon (Jatropha curcas). La Lima - Honduras. (2010).

A.O.A.C., Official Methods of Analysis, Association of Official Agriculture Chemists. 11th ed. USA. (1990).

A.O.C.S., American Oil Chemists' Society. Official method Cd 3d - 63. (2005).

Arruda, F.P.; Beltrão, N.E.M.; Andrade, A.P.; Pereira, W.E.; Severino, L.S., Revista Brasileira de Oleaginosas e Fibrosas, 8, 789. (2004) 
Frauches dos Santos C., Pires M. F. J., Faria-Machado A.F., Couto L., Kalaoum J., Correa D. Jr. P. Antoniassi R., Avaliação do rendimento de óleo no amadurecimento de frutos de pinhão-manso. II Congresso brasileiro de pesquisas de pinhão-manso. Brasilia - Brasil (2011).

Mejía, F., Cultivo de Jatropha curcas y construcción de una planta de biodiesel en San Esteban, Olancho, Honduras. (2006).

Rijssenbeek, W.; Galema T., Manual de Jatropha. Pag 43; Fuel From Agriculture in Communal Technology - FACT. Holanda. (2009).

Saturnino, H.M.; Pacheco, D.D.; Kakida, J.; Tominaga, N.; Gonçalves, N.P. Informe Agropecuário 229, 44. Brasil. (2005).

Vignoni L., Césari R., Forte M y Mirábile M. Determinación de Índice de Color en Ajo Picado. Información Tecnológica-Vol. 17 №6, pág.: 63-67 (2006). 
